\title{
BMJ Open Quality Using positive deviance to improve timely access in primary care
}

To cite: DeRocher M, Davie S, Kiran T. Using positive deviance to improve timely access in primary care. BMJ Open Quality 2021;10:e001228. doi:10.1136/ bmjoq-2020-001228

- Additional supplemental material is published online only. To view, please visit the journal online (http://dx.doi.org/10. 1136/bmjoq-2020-001228).

Received 19 0ctober 2020 Accepted 10 September 2021

Check for updates

(c) Author(s) (or their employer(s)) 2021. Re-use permitted under CC BY-NC. No commercial re-use. See rights and permissions. Published by BMJ.

${ }^{1}$ Department of Family and Community Medicine, St Michael's Hospital, Toronto, Ontario, Canada

${ }^{2}$ Department of Family and Community Medicine, University of Toronto, Toronto, Ontario, Canada

${ }^{3}$ MAP Centre for Urban Health Solutions, Li Ka Shing

Knowledge Institute, St.

Michael's Hospital, Toronto,

Ontario, Canada

${ }^{4}$ Institute of Health Policy,

Management, and Evaluation,

University of Toronto, Toronto,

Ontario, Canada

Correspondence to Dr MaryBeth DeRocher; marybeth.derocher@ unityhealth.to

MaryBeth DeRocher, ${ }^{1,2}$ Sam Davie, ${ }^{1}$ Tara Kiran (1) 1,2,3,4

\section{ABSTRACT}

Background Improving timely access in primary care is a continued challenge in many countries. We used positive deviance to try and identify best practices for achieving timely access in our primary care organisation in Toronto, Canada.

Methods Semistructured interviews were used to identify practice strategies used by physicians who successfully maintained a low third next available appointment (TNA) (positive deviants, $n=6$ ). We then conducted a crosssectional survey to understand the prevalence of identified promising practices among all physicians $(n=70)$ in the practice. We used $\chi^{2}$ testing to understand whether uptake of promising practices among survey respondents was different for those with a median TNA of 7 days or less vs a median TNA over 7 days.

Results We identified seven promising practice strategies used by positive deviants: adjusting the appointment template based on demand; reviewing the appointment schedule in advance; max-packing of visits; using phone, email and secure messaging; customising care for complex patients; managing planned absences; and involving the interprofessional team. 65 of 70 physicians responded to the survey on promising practices. Uptake of the promising practices was variable among survey respondents. In general, we found no association between uptake of promising practices and median TNA. One exception was that those with a median TNA of 7 or less were more likely to review the schedule in advance to potentially mitigate a visit using phone/email ( $62 \%$ vs $31 \%, p=0.0159$ ).

Conclusion Promising practices used by a small group of physicians ('positive deviants') to maintain good access were generally not associated with timely access among a larger sample of physicians in the practice. Our findings highlight the difficulty of untangling physician practice style and its contribution to timely access in primary care.

\section{INTRODUCTION}

Timely access is critical to achieving first contact and continuous care-two of the four pillars of primary care defined by Barbara Starfield three decades ago. ${ }^{1}$ Yet timely access remains a challenge in many countries, including both Canada and the USA, where only $41 \%$ and $48 \%$ of patients, respectively, report they could see their primary care provider on the same day or the next day when sick and needing care. ${ }^{2}$ Experts suggest that the problem is not lack of system capacity but rather system design. ${ }^{3}$
The Advanced access philosophy was considered a promising strategy to improve timely access, ${ }^{4}$ but real-world implementation has been difficult and improvements in waittimes modest. $^{5}$

For the last few years, our large primary care organisation has tried to measure and improve access to booked physician appointments as part of a broader strategy to improve timely access. We have routinely collected and fed back to individual physicians their third next available appointment (TNA), a common and accepted measure of timely access in primary care. ${ }^{67}$ The TNA is the number of calendar days from the time a patient requests an appointment with a physician to the third open appointment in the schedule. It captures the patient perspective of delay when seeking an appointment. The third open appointment is seen as a more reliable measure of system availability than the first or second as the latter may be related to a last-minute cancellation or other unpredictable event. In addition to reporting TNA, we offered physicians personalised coaching to improve using elements of the advanced access philosophy. Despite these efforts, we noted a persistently large variation in TNA between physicians. We also found no relationship between TNA and panel size, time in clinic or the ratio of panel size to time in the clinic, suggesting physician practice style was a critical driver of access. ${ }^{8}$

We sought to take a fresh approach to improving timely access in our primary care organisation using positive deviance. Positive deviance seeks to identify individuals, organisations and communities that have exceptionally good performance and learn from and spread their approaches. ${ }^{9}$ Positive deviance has its roots in public health, but its application in healthcare quality is relatively new and untested in primary care. ${ }^{10}$ We aimed to identify practice strategies used by family physicians who successfully maintained timely access to booked physician appointments and test whether these strategies were correlated with timely access among all family physicians in the organisation. 
Step 1: Identify "Positive Deviants"

Approach individuals who consistently

demonstrated low Third Next Available

Appointment (TNA) in the preceding year
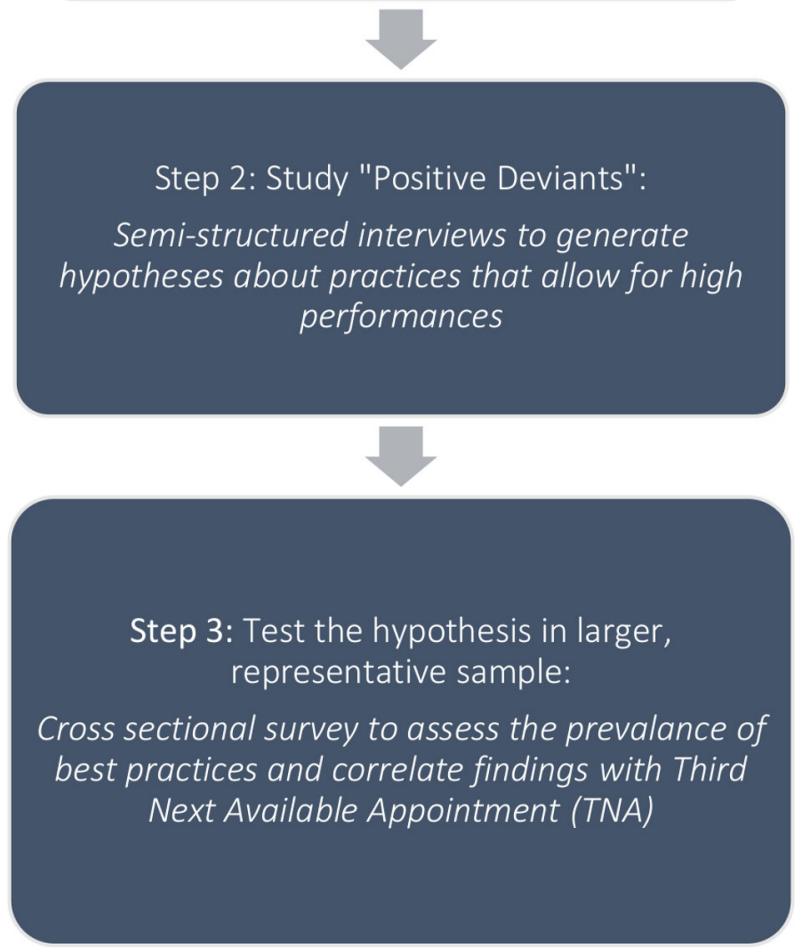

Figure 1 Steps in the positive deviance approach. Modified from Lawton et al. ${ }^{10}$

\section{METHODS}

\section{Setting and context}

The St. Michael's Academic Family Health Team is a primary care organisation that serves over 49000 enrolled patients at six clinics in downtown Toronto, Canada. At the time of study, the team included 70 staff physicians, 40 resident physicians and over 60 non-physician health professionals. Since 2015, TNA has been collected biweekly through manual audit of staff physicians' appointment schedule with individual measures fed back to staff physicians in real time via email. Day of collection is rotated and data are maintained in a Microsoft Access database. Between May 2016 and April 2017, the mean TNA for staff physicians was 10.5 days $(\mathrm{n}=74, \mathrm{SD}=10.3$, range $=0-74$ ). The mean roster size was 577 patients and staff averaged 5.5 days in clinic. At the time of the initiative, patients could book clinic appointments by phone or in person.

\section{Study design}

We used a mixed-methods approach based on the positive deviance methodology outlined by Lawton and colleagues $^{10}$ (figure 1). The term 'positive deviance' was first coined in public health and defined as 'the observation that in most settings a few at risk individuals follow uncommon, beneficial practices and consequently experience better outcomes than their neighbours who share similar risks'. Public health programmes have historically used this approach to identify and spread beneficial practices in a community to, for example, improve the nutritional status of children. More recently, the positive deviance approach has been used successfully in healthcare to drive improvements on topics ranging from hand hygiene ${ }^{11}$ to care for people with diabetes. ${ }^{12}$ At its core, positive deviance hinges on systematically identifying individuals, teams or organisations with exemplary performance, learning from their practices and context, and then spreading what was learnt to others. ${ }^{10}$ The premise is that solutions to complex problems lie within the tacit knowledge and wisdom of the clinical communities where the problem exists rather than with managers or policy makers.

The first step in the methodology is to identify the positive deviants-individuals, teams or organisations with exemplary performance. These positive deviants are then studied using in-depth qualitative methods to generate hypotheses about potential practices that lead to high performance. Next, the hypotheses are tested among a larger, representative sample. Finally, the newly identified best practices are spread. We adapted this approach for our primary care setting: we identified physicians with consistently low TNA (positive deviants). We used semistructured individual interviews to identify promising practices related to the low TNA. We conducted a crosssectional survey among family physicians in the group to understand uptake of these promising practices. Finally, we correlated whether there was a correlation between the promising practices and TNA.

\section{Identification of positive deviants}

We identified six staff physicians with consistently low TNA in the year prior to September 2016 (positive deviants; median annual TNA ranged from 2 to 5). We aimed to identify individuals with diversity in clinical site, size of practice, time in clinic, age and gender.

\section{Semistructured interviews to identify promising practice strategies}

We asked physicians in our organisation identified as positive deviants to participate in semistructured qualitative interviews and all agreed. All physicians were interviewed between September 2016 and January 2017 by the lead author (MDR), a practising physician in the department. The interview probed for strategies used to maintain timely access to booked appointments, use of specific strategies such as adoption of e-communication and use of the interprofessional team, and barriers to maintaining timely access. The interview also probed for a sense of provider values regarding access. The interviewer used handwritten notes to record responses, which were transcribed into typed format following the interview. The 
study team used thematic analysis ${ }^{13}$ to analyse responses iteratively coding the data and then identifying themes and related practice strategies.

\section{Cross-sectional survey to understand physician practice patterns}

We developed a survey to assess the prevalence of promising practices identified in the semistructured interviews and physician attitudes towards timely access (online supplemental appendix 1). The survey was hosted on Qualtrics and administered via email to all staff physicians $(n=70)$ in April 2017. Staff physicians received a unique survey link and up to two individual reminders over a 6-week period. We conducted a descriptive analysis of survey data to understand the proportion of physicians reporting they never, rarely, sometimes, often or always used a strategy. All surveys were included in the analysis.

\section{Correlation of promising practices with physician TNA}

We calculated physicians' median TNA during the period from May 2016 to April 2017. We then performed two types of analyses to assess the association between adoption of promising practices and physicians' median TNA. In the first analysis, we stratified physicians using TNA quintiles (quintile 1=lowest median TNA; quintile 5 =highest median TNA). For each TNA quintile, we calculated the proportion of physicians who reported always or often using a practice strategy and looked for crude associations. Due to small cells, we did not assess for statistical significance when data were stratified by TNA quintiles.

The second analysis dichotomised physicians based on whether their median TNA was (1) 7 days or less or (2) over 7 days. For each group, we calculated the proportion of physicians who reported always or often using a practice strategy. We then used $\chi 2$ testing (and Fischer's exact test for cell size $<5$ ) to assess whether observed differences were statistically significant. All analyses were conducted in SAS V.9.

\section{Patient and public involvement}

Our team's focus on improving timely access was driven by feedback from our patient experience survey. Although patients were not involved in the design or conduct of this study, we will be sharing and discussing our findings with our patient partners to inform future work in our practice to improve access.

\section{RESULTS}

\section{Semistructured interviews with positive deviants}

We interviewed six physicians with consistently low TNA ('positive deviants'). Interviews revealed seven themes and related practice strategies used to support timely access to booked appointments: (1) adjusting the appointment template based on demand; (2) reviewing the appointment schedule in advance; (3) max-packing of visits; (4) using phone, email or secure messaging; (5) customising care for complex patients; (6) managing planned absences; and (7) involving the interprofessional team (table 1).

The following strategies were used by most or all of the positive deviants. First, most positive deviants noted that they would add new appointment slots based on when their reported TNA increased. Second, all had approaches for managing planned absences, including informing patients in advance of their absence, ensuring appropriate coverage through practice sharing or collaborating with clerical staff to limit non-urgent appointments (eg, routine diabetes reviews, well-baby visits and period health visits) until a week or two after their return to clinic. Finally, most collaborated with non-physician team members to improve timely access, deliberately sharing the care to free up time in their own schedule.

Interviews also revealed some differences in approaches to maintaining timely access. Some began their day as early as possible to maximise their availability, while others mentioned that it was important to have administrative time at the start of the day to deal with urgent issues. Some physicians identified electronic communication with patients (email or secure messaging) as an important tool for providing care, even when outside of the office, while others felt that this strategy was not required (or desired) to support timely access.

\section{Cross-sectional survey to understand physician practice patterns}

Sixty five of seventy staff physicians (93\%) responded to the survey assessing practice patterns. Among respondents, the average years since graduation was 17.1 years (median=13 years) and $62 \%$ were female.

Most physicians reported always or often using strategies related to max-packing, use of faxed prescription renewals, spreading clinics across the week to maximise availability, use of proactive preventative health manoeuvres within an opportunistic visit and involving the interdisciplinary team for immunisations (table 1). There was variation in most other areas of practice.

The association between practice strategies and TNA quintile is summarised in figure 2. Stratified analysis did not show higher uptake of strategies in quintile 1 (median $\mathrm{TNA}=3$ days) compared with quintile 5 (median TNA=15 days) with three exceptions. Compared with TNA quintile 5, more physicians in TNA quintile one reported adjusting their clinic schedule based on TNA $(31 \%$ vs $15 \%$ ), adjusting the number of same/next-day slots based on demand ( $31 \%$ vs $15 \%)$ and reviewing the schedule in advance to potentially mitigate a visit using phone or email $(69 \%$ vs $39 \%)$.

Results were similar when physicians were dichotomised by TNA. There were no statistically significant differences in best practice uptake with one exception: those with a median TNA of 7 or less were more likely to review the schedule in advance to potentially mitigate a visit using phone or email $(62 \%$ vs $31 \%, \mathrm{p}=0.0159)$ compared with those with a TNA of less than 7 (online supplemental appendix 2). 
Table 1 Practice strategies used by physicians with consistently low TNA

Practice strategies used by physicians with consistently low TNA

Percentage of staff physicians reporting they 'always' or 'often' use the strategy on departmentwide survey $(n=65)$

\section{Theme}

\section{Specific strategies}

$23 \%$

Adjusting the appointment template based on demand

Adjusting clinic schedule based on TNA*

Starting morning clinic before 09:00

$22 \%$

Using administrative slots at the start of clinic to accommodate urgent visits $\quad 18 \%$

Scheduling clinic half days to be spread out across the week $^{*} \quad 92 \%$

Adjusting number of same/next-day slots based on patient demand $\quad 18 \%$

Educating patients on use of same/next-day slots*

Reviewing the appointment schedule in advance

Reviewing to ensure booked patients are part of roster*

$42 \%$

Ensuring that patients are not booked with more than one appointment ${ }^{\star} \quad 37 \%$

Reviewing to see if email/call to patient directly can mitigate a visit $46 \%$

Ensuring that test results have come in which are necessary for review in the $54 \%$ upcoming visit

Ensuring that patient does not need an earlier appointment for an urgent issue ${ }^{\star} \quad 42 \%$

Ensuring that a longer appointment is not required for a more urgent concern $\quad 57 \%$

\begin{tabular}{|c|c|c|}
\hline \multirow[t]{3}{*}{ Max-packing of visits } & Using fax prescription renewals* & $95 \%$ \\
\hline & Dealing with multiple problems in one visit if the patient brings them up* & $98 \%$ \\
\hline & $\begin{array}{l}\text { Proactively addressing multiple patient issues in a single visit to avoid another visit (ie, } \\
\text { max-pack) })^{\star}\end{array}$ & $89 \%$ \\
\hline \multirow{3}{*}{$\begin{array}{l}\text { Using phone, email and/or secure } \\
\text { messaging }\end{array}$} & Communicating with patients via email or secure messaging for clinical issues & $25 \%$ \\
\hline & Integrating phone appointments with patients into my regular clinic & $14 \%$ \\
\hline & $\begin{array}{l}\text { Communicating test results with patients using email, secure messaging, phone or } \\
\text { mailed letter }\end{array}$ & $63 \%$ \\
\hline \multirow[t]{2}{*}{ Managing complex patients } & Booking longer appointments for complex patients* & $52 \%$ \\
\hline & $\begin{array}{l}\text { Adjusting the time between follow-up appointments based on the disease stability for } \\
\text { complex patients }{ }^{\star}\end{array}$ & $86 \%$ \\
\hline & Avoiding taking vacations during typically busy periods & $28 \%$ \\
\hline & Using postvacation blocking & $63 \%$ \\
\hline \multirow[t]{7}{*}{ Involving the interdisciplinary team } & Using non-physician team members to help with well-baby checks* & $81 \%$ \\
\hline & Using non-physician team members to help with immunisations & $91 \%$ \\
\hline & Using non-physician team members to help with hypertension follow-up & $44 \%$ \\
\hline & Using non-physician team members to help with cancer screening & $5 \%$ \\
\hline & Using non-physician team member to help with reminder calls & $53 \%$ \\
\hline & Using non-physician team members to help with communication of test results & $59 \%$ \\
\hline & Using non-physician team members to help with preventative health exams & $52 \%$ \\
\hline
\end{tabular}

*Denotes strategies used by all interviewed physicians.

\section{DISCUSSION}

We identified several practice strategies used by a small number of physicians who successfully maintained relatively short waits for booked appointments-our positive deviants. Promising practice strategies included adjusting the appointment template based on demand, reviewing the appointment schedule in advance, max-packing of visits, using phone, email or secure messaging, customising care for complex patients, managing planned absences and involving the interprofessional team. However, empiric testing in a larger sample of physicians in our practice found that there was, in general, no difference in the uptake of these strategies among physicians with longer versus shorter waits for booked appointments as measured by median TNA.

The promising practice strategies we identified from positive deviants are consistent with those recommended by quality improvement experts as approaches 
A Managing Planned Absences

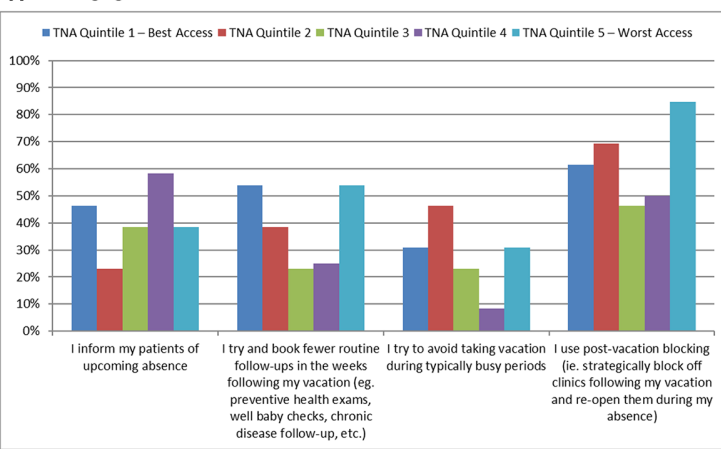

C Using Phone, Email, and Secure Messaging

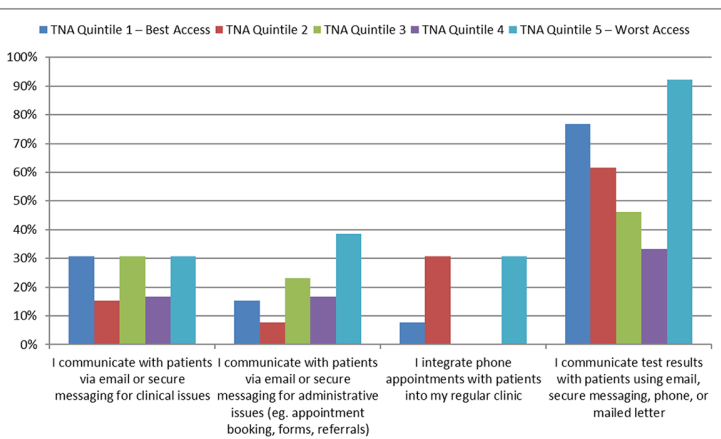

E Max-Packing of Visits

- TNA Quintile 1 - Best Access $\boldsymbol{m}$ TNA Quintile 2 = TNA Quintile 3 m TNA Quintile 4 m TNA Quintile 5 - Worst Access

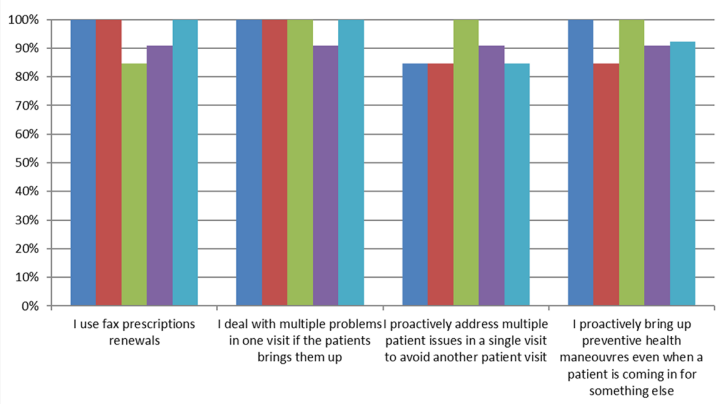

B Customizing Care for Complex Patients

- TNA Quintile 1- Best Access = TNA Quintile 2 m TNA Quintile 3 m TNA Quintile 4 m TNA Quintile 5- Worst Access

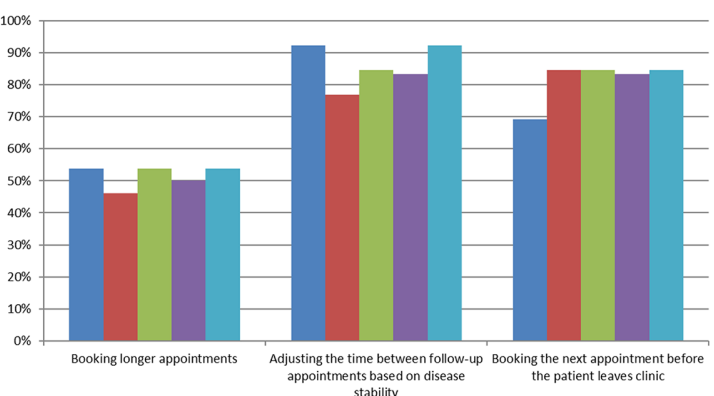

D Adjusting Appointment Based upon Demand

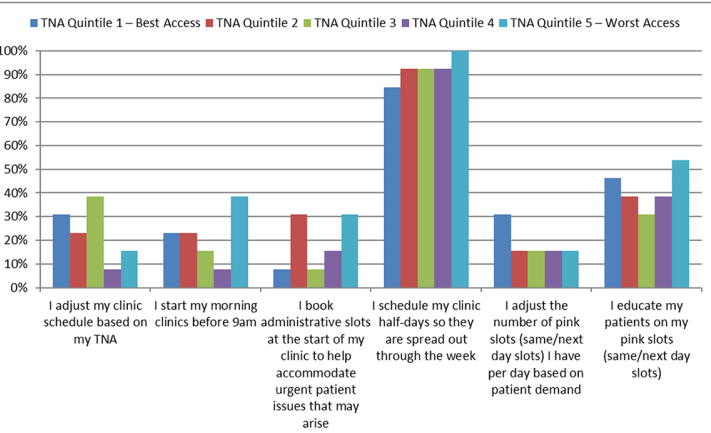

F Involving the Interprofessional Team

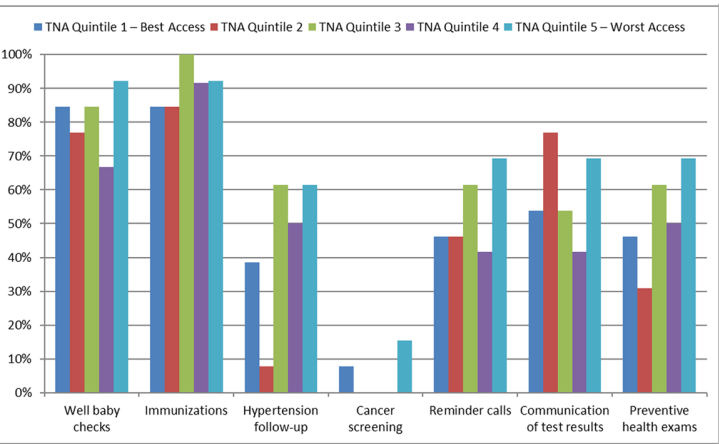

F Reviewing the Appointment Schedule in Advance

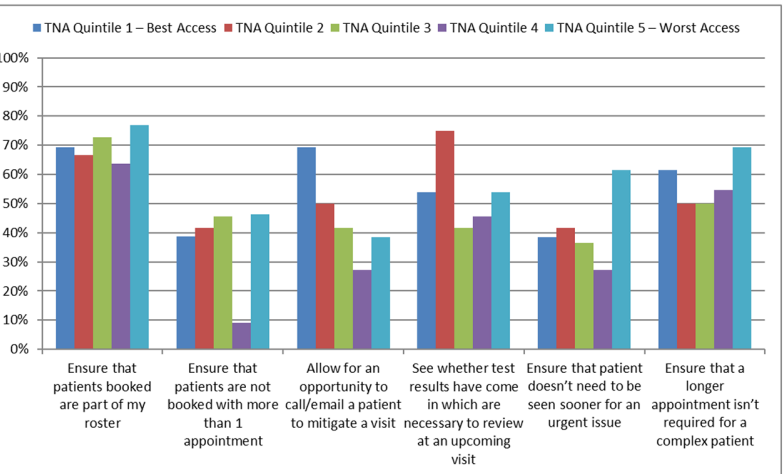

Figure 2 Uptake of practice strategies among staff physicians stratified by TNA quintile. (A) Managing planned absences. (B) Customising care for complex patients. (C) Using phone, email, and secure messaging. (D) Adjusting appointment based on demand. (E) Max-packing of visits. (F) Involving the interprofessional team. (G) Reviewing the appointment schedule in advance.

to improve timely access and are consistent with principles used in advanced access to have supply of appointments meet demand. ${ }^{14}$ This consistency is not surprising, given our practices efforts to educate physicians about advanced access in the years prior to the study. Our cross-sectional study design may partly explain why we found no association between uptake of the strategies and TNA quintile. It is possible that 
physicians with high TNA have seen an improvement in their waits over time with adoption of the strategiessomething we were not able to assess. Our study also only focused on understanding reasons behind physician variation, and it is possible that timeliness would be more consistently improved by more systematically involving clerical staff in improvement efforts.

We did find a correlation between TNA and three practice strategies. Physicians with lower median TNA were more likely to adjust their schedule based on TNA and adjust their same/next-day slots based on patient demand. This correlation was not statistically significant but may reflect the value of TNA feedback for physicians who accept and use the feedback. Physicians with lower median TNA were more likely to use telephone or email to mitigate an office visit. This finding is consistent with research from other jurisdictions noting improvements in timely access with adoption of e-communication. ${ }^{15}{ }^{16}$ Prior to COVID-19, e-communication between physicians and patients was relatively uncommon in Canada despite its appeal to patients, ${ }^{17}$ and practice and policy changes are needed to further increase and sustain their use.

Our study has several strengths. We used a unique approach-positive deviance-to identify practical strategies physicians could adopt to improve timely access. We had a high response rate to our physician survey and were able to leverage routine collection of TNA in our practice setting, a unique data holding. However, our study also has limitations. First, we relied on physicians to self-report practice strategies. Second, our study was observational and cross-sectional and so cannot infer causation; physicians with higher TNA may have used some of the identified strategies to improve over time. Third, our study was conducted in a large interprofessional primary care organisation engaged in improving timely access for a few years; the setting may limit generalisability of some findings (eg, promising practices) but not our positive deviance approach. Finally, our study relied on TNA as a measure of access to booked physician appointments-a measure accepted in the literature but sometimes questioned by physicians in our organisation.

Our findings highlight the variation in individual physician practice, even within a single organisation, and the known challenge of improving timely access to booked physician appointments. Although we identified several promising strategies used by positive deviants, physician practice style may be too complex to break down into component parts. Instead, we are now trialling mentorship by positive deviants, allowing physicians to choose mentors that most closely reflect their own practice style. We are also working to strengthen the building blocks for achieving access including engaged leadership, datadriven improvement and team-based care ${ }^{18}$ Our survey of physicians revealed variation within and across our clinics in how physicians collaborate with other health professionals to share the care-a known strategy to improve access. ${ }^{19}$ Finally, we recognise the inherent limitations of TNA as a measure of access and have now moved to collecting and confidentially reporting physician-level indicators of access from our patient experience survey.

\section{CONCLUSION}

We used positive deviance to identify promising practice strategies among physicians who consistently maintained timely access to booked appointments as measured by TNA. These promising practice strategies were consistent with known expert advice but were generally not associated with timely access among a larger sample of physicians in the practice. Our results reflect the difficulty of untangling physician practice style and its contribution to timely access in primary care. Twitter Tara Kiran @tara_kiran

Acknowledgements We thank Amy McDougall for her contributions to data collection and insights; Chris Meaney and Rahim Moineddin for their statistical advice; and our colleagues who participated in this initiative and strive to make our clinics more accessible.

Contributors All authors conceived of the initiative, designed the study, were involved in data collection, and interpreted the findings. SD conducted the quantitative analysis. MDR and TK wrote the first draft of the manuscript and all authors critically reviewed it. All authors read and approved the final manuscript.

Funding This study received no direct funding. TK is supported as a clinician scientist by the Department of Family and Community Medicine at the University of Toronto and at St. Michael's Hospital. She is the Fidani Chair in Improvement and Innovation at the University of Toronto. At the time of writing, she was also supported by the Canadian Institutes of Health Research and Health Quality Ontario as an embedded clinician researcher. The opinions, results and conclusions reported in this article are those of the authors and are independent from the funding sources

\section{Competing interests None declared.}

Patient consent for publication Not applicable.

Ethics approval This initiative was formally reviewed by institutional authorities at St. Michael's Hospital and deemed to neither require research ethics board approval nor written informed consent from participants.

Provenance and peer review Not commissioned; externally peer reviewed.

Data availability statement All data relevant to the study are included in the article or uploaded as supplementary information. De-identified participant data is available on request from the corresponding author.

Supplemental material This content has been supplied by the author(s). It has not been vetted by BMJ Publishing Group Limited (BMJ) and may not have been peer-reviewed. Any opinions or recommendations discussed are solely those of the author(s) and are not endorsed by BMJ. BMJ disclaims all liability and responsibility arising from any reliance placed on the content. Where the content includes any translated material, BMJ does not warrant the accuracy and reliability of the translations (including but not limited to local regulations, clinical guidelines, terminology, drug names and drug dosages), and is not responsible for any error and/or omissions arising from translation and adaptation or otherwise.

Open access This is an open access article distributed in accordance with the Creative Commons Attribution Non Commercial (CC BY-NC 4.0) license, which permits others to distribute, remix, adapt, build upon this work non-commercially, and license their derivative works on different terms, provided the original work is properly cited, appropriate credit is given, any changes made indicated, and the use is non-commercial. See: http://creativecommons.org/licenses/by-nc/4.0/.

ORCID iD

Tara Kiran http://orcid.org/0000-0002-2520-112X

\section{REFERENCES}

1 Starfield B. Primary care: concept, evaluation, and policy. Oxford University Press, 1992. 
2 Robin O, Schoen C. Chartpack: the Commonwealth fund 2013 international health policy survey in eleven countries. Commonwealth Fund 2013.

3 Murray M, Tantau C. Same-Day appointments: exploding the access paradigm. Fam Pract Manag 2000;7:45-9.

4 Murray M, Berwick DM. Advanced access. JAMA 2003;289:1035-40.

5 Rose KD, Ross JS, Horwitz LI. Advanced access scheduling outcomes: a systematic review. Arch Intern Med 2011;171:1150.

6 Institute for Healthcare Improvement. Third next available appointment, 2018. Available: http://www.ihi.org/resources/Pages/ Measures/ThirdNextAvailableAppointment.aspx

7 US Department of Veterans Affairs, Health Services Research \& Development Service. Access management improvement: a systematic review. Available: https://www.hsrd.research. va.gov/ publications/esp/AccessMgt-REPORT.pdf [Accessed Jul 2021].

8 Derocher M, Alhaj M, Kiran T. No easy fix to improve timely access to booked appointments [Letter to the editor]. Annals of Family Medicine 2018 http://www.annfammed.org/content/16/6/546.short/ reply - annalsfm_el_30811

9 Marsh DR, Schroeder DG, Dearden KA, et al. The power of positive deviance. BMJ 2004:329:1177-9.

10 Lawton R, Taylor N, Clay-Williams R, et al. Positive deviance: a different approach to achieving patient safety. BMJ Qual Saf 2014;23:880-3.
11 Marra AR, Guastelli LR, de Araújo CMP, et al. Positive deviance: a new strategy for improving hand hygiene compliance. Infect Control Hosp Epidemiol 2010;31:12-20.

12 Gabbay RA, Friedberg MW, Miller-Day M, et al. A positive deviance approach to understanding key features to improving diabetes care in the medical home. Ann Fam Med 2013;11 Suppl 1:S99-107.

13 Saldaña J. The coding manual for qualitative researchers. Sage, 2015.

14 Murray M, Tantau C. Redefining open access to primary care. Manag Care Q 1999;7:45-55.

15 Reid RJ, Coleman K, Johnson EA, et al. The group health medical home at year two: cost savings, higher patient satisfaction, and less burnout for providers. Health Aff 2010;29:835-43.

16 Bishop TF, Press MJ, Mendelsohn JL, et al. Electronic communication improves access, but barriers to its widespread adoption remain. Health Aff 2013;32:1361-7.

17 Carroll JC, Talbot Y, Permaul J, et al. Academic family health teams: Part 2: patient perceptions of access. Can Fam Physician 2016;62:e31-9.

18 Bodenheimer T, Ghorob A, Willard-Grace R, et al. The 10 building blocks of high-performing primary care. Ann Fam Med 2014;12:166-71.

19 Sinsky CA, Willard-Grace R, Schutzbank AM, et al. In search of joy in practice: a report of 23 high-functioning primary care practices. Ann Fam Med 2013;11:272-8. 